\title{
Molecular and Structural Basis of the Proteasome $\alpha$ Subunit Assembly Mechanism Mediated by the Proteasome-Assembling Chaperone PAC3-PAC4 Heterodimer
}

\author{
Tadashi Satoh ${ }^{1}$, Maho Yagi-Utsumi ${ }^{1,2,3}$, Kenta Okamoto ${ }^{1}$, Eiji Kurimoto ${ }^{4}$, Keiji Tanaka ${ }^{5}$ \\ and Koichi Kato $1,2,3, *$ iD \\ 1 Graduate School of Pharmaceutical Sciences, Nagoya City University, 3-1 Tanabe-dori, Mizuho-ku, \\ Nagoya 467-8603, Japan; tadashisatoh@phar.nagoya-cu.ac.jp (T.S.); mahoyagi@ims.ac.jp (M.Y.-U.); \\ kenta.okamoto@icm.uu.se (K.O.) \\ 2 Exploratory Research Center on Life and Living Systems (ExCELLS), National Institutes of Natural Sciences, \\ 5-1 Higashiyama, Myodaiji, Okazaki, Aichi 444-8787, Japan \\ 3 Institute for Molecular Science, National Institutes of Natural Sciences, 5-1 Higashiyama, Myodaiji, \\ Okazaki, Aichi 444-8787, Japan \\ 4 Faculty of Pharmacy, Meijo University, Tempaku-ku, Nagoya 468-8503, Japan; kurimoto@meijo-u.ac.jp \\ 5 Laboratory of Protein Metabolism, Tokyo Metropolitan Institute of Medical Science, 2-1-6, Kamikitazawa, \\ Setagaya-ku, Tokyo 156-8506, Japan; tanaka-kj@igakuken.or.jp \\ * Correspondence: kkatonmr@ims.ac.jp; Tel.: +81-564-59-5225; Fax: +81-564-59-5224
}

Received: 27 March 2019; Accepted: 3 May 2019; Published: 7 May 2019

\begin{abstract}
The 26S proteasome is critical for the selective degradation of proteins in eukaryotic cells. This enzyme complex is composed of approximately 70 subunits, including the structurally homologous proteins $\alpha 1-\alpha 7$, which combine to form heptameric rings. The correct arrangement of these $\alpha$ subunits is essential for the function of the proteasome, but their assembly does not occur autonomously. Assembly of the $\alpha$ subunit is assisted by several chaperones, including the PAC3-PAC4 heterodimer. In this study we showed that the PAC3-PAC4 heterodimer functions as a molecular matchmaker, stabilizing the $\alpha 4-\alpha 5-\alpha 6$ subcomplex during the assembly of the $\alpha$-ring. We solved a $0.96-\AA$ atomic resolution crystal structure for a PAC3 homodimer which, in conjunction with nuclear magnetic resonance (NMR) data, highlighted the mobility of the loop comprised of residues 51 to 61 . Based on these structural and dynamic data, we created a three-dimensional model of the PAC $3-4 / \alpha 4 / \alpha 5 / \alpha 6$ quintet complex, and used this model to investigate the molecular and structural basis of the mechanism of proteasome $\alpha$ subunit assembly, as mediated by the PAC3-PAC4 heterodimeric chaperone. Our results provide a potential basis for the development of selective inhibitors against proteasome biogenesis.
\end{abstract}

Keywords: assembly chaperone; molecular matchmaker; molecular modeling; proteasome; X-ray crystal structure

\section{Introduction}

The selective degradation of proteins in eukaryotic cells is essential for the maintenance of physiological homeostasis. Protein degradation is implemented primarily via the ubiquitin-proteasome system [1,2]. The proteasome is huge protein complex (26S), comprised of a $20 \mathrm{~S}$ core particle (CP) and one or two $19 \mathrm{~S}$ regulatory particles (RPs). The $20 \mathrm{~S} \mathrm{CP}$, which has proteolytic activity, is composed of seven homologous $\alpha$ subunits, $\alpha 1-\alpha 7$, and seven homologous $\beta$ subunits, $\beta 1-\beta 7$, which are assembled into a cylindrical structure with an $\alpha_{1-7} \beta_{1-7} \beta_{1-7} \alpha_{1-7}$ arrangement. The 19S RP is responsible for the 
collection of ubiquitinated substrates, the opening of the central gating pore of the $20 \mathrm{SCP}$, and the de-ubiquitination and translocation-coupled unfolding of the substrates. Recent structural studies using cryo-electron microscopy have shed light upon the cooperative working mechanisms of this huge proteolytic machinery [3,4].

The correct arrangement of the proteasomal subunits is essential to the proper functioning of eukaryotic proteasomes. There is considerable evidence that the assembly of the eukaryotic $26 \mathrm{~S}$ proteasome does not proceed spontaneously, but is mediated by several assembly chaperones [5-8]. The formation of the 20S CP is assisted by five proteasome-specific chaperones: PAC1-PAC4 and POMP in humans; Pba1-Pba4 and Ump1 in yeast. Four dedicated chaperones, p27 (Nas2), gankyrin (Nas6), PAAF1 (Rpn14), and S5b (Hsm3) are responsible for the formation of the base subcomplex of the 19S RP. Malfunctions of these assembly chaperones cause the accumulation of imperfectly assembled or mis-assembled complexes of the proteasomal subunits. For example, knock-down experiments involving PAC 3 and PAC4 results in the accumulation of abnormal $\alpha$-subunit oligomers lacking the $\alpha 3-\alpha 7$ subunits $[9,10]$.

Because proteasome biogenesis is known to be significantly upregulated in cancer cells [11], the proteasome has potential as a target for therapeutic drugs for cancer treatment $[12,13]$. Bortezomib (Velcade) has been widely used as proteasome inhibitor for the treatment of patients with multiple myeloma [14]. The chaperones involved in proteasome assembly have also been considered as potential drug targets for anticancer treatments [12]. Selective inhibitors that specifically suppress proteasome biogenesis could be valuable for minimizing the undesired side effects which can occur when using compounds which target mature proteasomes.

Recently reported knock-out experiments indicated that $\alpha 4, \alpha 5, \alpha 6$, and $\alpha 7$ form a core assembly intermediate as part of the initial process of $\alpha$-ring assembly, which is supported by PAC3-PAC4 [15]. However, most of the biochemical and structural data about the proteasome-assembly chaperones have been generated mainly from yeast proteins, which have only modest sequence identities with the human counterparts; less than $20 \%$, for PAC3 when compared with Pba3. As with CP-assembly, yeast $\mathrm{Pba} 3$ and $\mathrm{Pba} 4$ have structural resemblance, and form a heterodimer [16] which functions as a matchmaker mediating the association between $\alpha 4$ and $\alpha 5$ [17]. It remains unclear, however, how the human PAC3-PAC4 complex functions in $\alpha$-ring assembly through specific, direct interactions with cognate proteasomal subunits, although the crystal structures of human PAC3 and PAC4 have been solved for their homodimeric forms $[16,18]$.

Structural insights into the chaperone-mediated formation of the human proteasome are important for the design and development of low-toxicity anticancer drugs which can inhibit the protein-protein interactions involved in the proteasome-assembly process. We performed a biochemical and biophysical study of the human PAC3-PAC4 heterodimer in order to understand the functional and structural mechanisms of $\alpha$-ring formation mediated by the proteasome-assembling chaperones.

\section{Results and Discussion}

\subsection{The PAC3-PAC4 Heterodimer Interacts Primarily with $\alpha 5$}

To study the biochemical processes involved in proteasome $\alpha$-subunit assembly mediated by the PAC3-PAC4 heterodimer, we prepared all of the human proteasome $\alpha$ subunits as recombinant proteins. Although protocols to prepare PAC3 and PAC4 as individual recombinant proteins have been reported previously, their heterodimer is rather unstable, unlike the yeast orthologs $\mathrm{Pba} 3$ and $\mathrm{Pba} 4[16,18]$. The recombinant PAC4 also has a tendency to form a domain-swapped homodimer [18]. To overcome these problems, we designed and prepared a PAC3-PAC4 heterodimer as a single-chain form, termed scPAC3/4, in which the C-terminus of PAC4 is connected to the $\mathrm{N}$-terminus of PAC3 via a (GGGS) $)_{4}$ liner. All of the recombinant proteins were produced using bacterial expression systems in Escherichia coli, and were successfully purified to homogeneity (Figure S1). 
To determine which proteasomal $\alpha$ subunits interact with the PAC3-PAC4 heterodimer, we performed in vitro pull-down experiments using these recombinant proteins. In the pull-down assay, His $_{6}$-tagged scPAC $3 / 4$ was applied to $\mathrm{Ni}^{2+}$-charged resin, and subsequently incubated with a mixture of all of the $\alpha$-subunit proteins. Since $\alpha 7$ spontaneously forms an oligomer that is capable of capturing $\alpha 6[19,20]$, we carried out this experiment both in the absence and in the presence of $\alpha 7$. The pull-down experiments showed that scPAC $3 / 4$ reacted with several $\alpha$ subunits including $\alpha 4, \alpha 5$, and $\alpha 6$ (Figure 1 ). Addition of $\alpha 7$ had virtually no impact on the interaction of $\alpha 6$ with scPAC $3 / 4$, suggesting that it has a higher affinity for the PAC3-PAC4 heterodimer than for the $\alpha 7$ oligomer. To avoid ambiguity due to overlapping of the Coomassie Brilliant Blue (CBB)-stained bands, we performed the pull-down experiments using $\alpha 1, \alpha 4, \alpha 5$, and $\alpha 6$ individually. The pull-down assay showed that scPAC $3 / 4$ interacted most strongly with $\alpha 5$ and weakly with $\alpha 4$ and $\alpha 6$. By contrast, no interaction was detected between scPAC3/4 and $\alpha 1$. The interaction between $\alpha 6$ and scPAC $3 / 4$ appeared to be enhanced in the presence of the other $\alpha$ subunits. Since $\alpha 5$ and $\alpha 6$ occur consecutively in the native $\alpha$ ring, these data suggest that the PAC3-PAC4 heterodimer is important for $\alpha 5-\alpha 6$ subcomplex assembly during proteasome $\alpha$-ring formation.

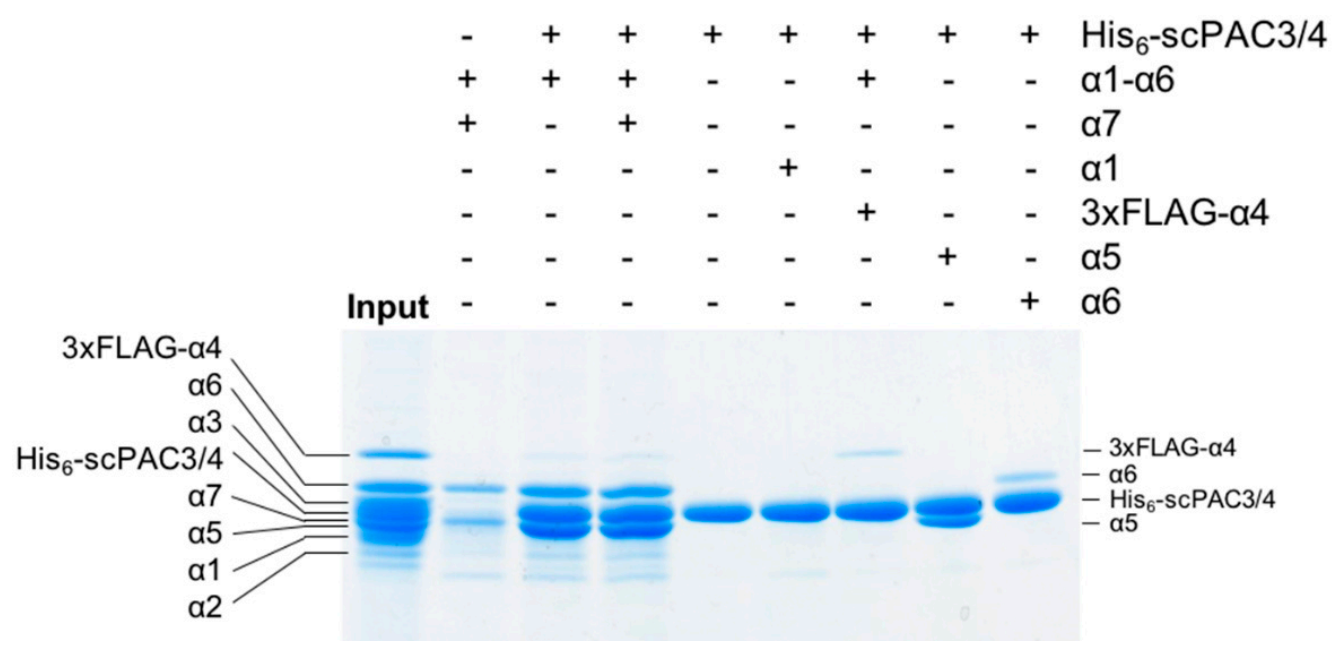

Figure 1. Pull-down experiments between the PAC3-PAC4 heterodimer and proteasome $\alpha$ subunits. The non-tagged $\alpha 1-\alpha 3$ and $\alpha 5-\alpha 7$ along with 3xFLAG-tagged $\alpha 4$ were mixed with His ${ }_{6}$-tagged scPAC3/4 immobilized on $\mathrm{Ni}^{2+}$-charged Chelating Sepharose beads. The 3xFLAG-tagged $\alpha 4$ was used to avoid the band overlap between $\alpha 4$ and scPAC3/4. After extensive washing, bound proteins were analyzed using CBB staining after sodium dodecyl sulfate polyacrylamide gel electrophoresis (SDS-PAGE). The 'Input' lane contained all $\alpha$ subunits and His $_{6}$-scPAC3/4 (0.5 $\mu \mathrm{g}$ each). The SDS-PAGE bands were assigned according to Figure S1a, and the bands originating from the $\mathrm{His}_{6}$-scPAC3/4 and the bound $\alpha$ subunits are labeled.

\subsection{The PAC3-PAC4 Heterodimer Acts as Molecular Matchmaker in $\alpha 4-\alpha 5-\alpha 6$ Assembly}

In order to explore the functional mechanism of the PAC3-PAC4 heterodimer in proteasome assembly involving $\alpha 4-\alpha 6$, we investigated the inter-subunit interactions mediated by scPAC $3 / 4$. In a pull-down assay, glutathione $S$-transferase (GST)-fused $\alpha 5$ was used as a bait for probing its interactions with the other $\alpha$ subunits, both in the absence and in the presence of scPAC $3 / 4$. GST- $\alpha 5$ interacted weakly with $\alpha 6$ in the presence of scPAC3/4, while the other subunits were not reactive with $\alpha 5$ regardless of the presence or absence of scPAC3/4 (Figure 2). In contrast, GST- $\alpha 5$ weakly interacted with $\alpha 4$ regardless of the presence or absence of scPAC3/4 under this assay condition. The results were not influenced by the presence of $\alpha 7$ (Figures 1 and 2). 
(a)

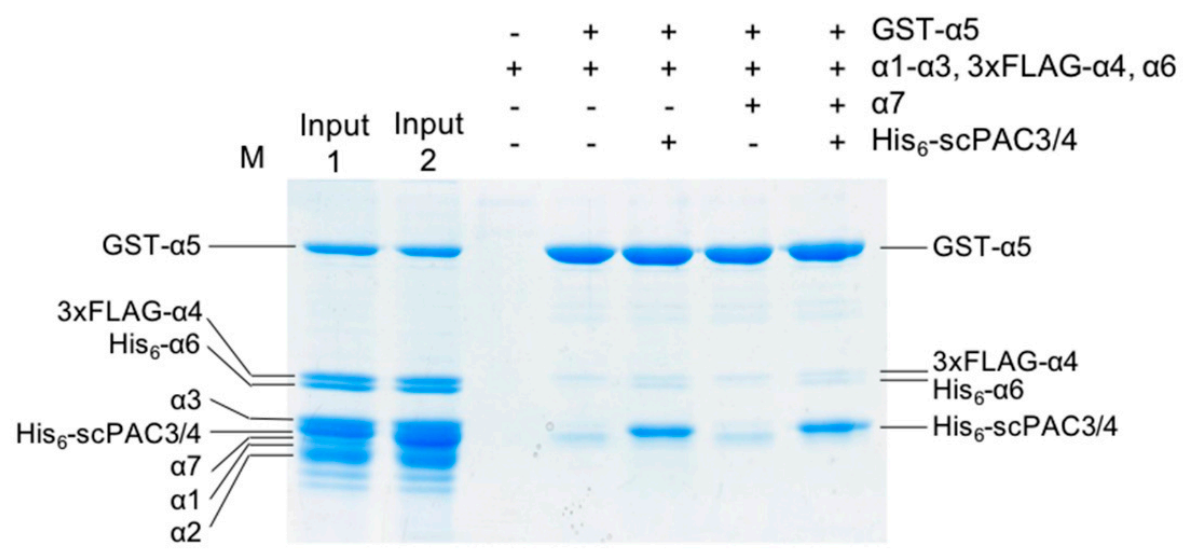

(b)

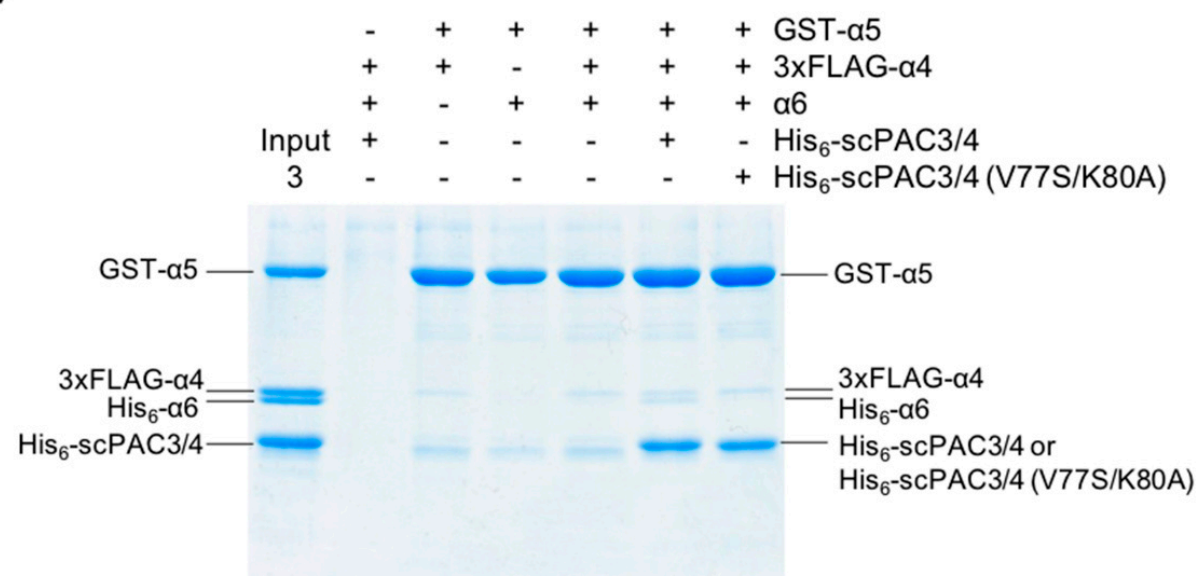

Figure 2. Pull-down experiments between $\alpha 5$ and the other $\alpha$ subunits. The $\alpha 1-\alpha 4, \alpha 6$, and $\alpha 7$ subunits were mixed with GST-tagged $\alpha 5$ immobilized on Glutathione Sepharose beads. The 3xFLAG-tagged $\alpha 4$ and His $_{6}$-tagged $\alpha 6$ were used to avoid the overlap of their bands with those of the other $\alpha$ subunits or scPAC3/4. (a) Interactions between $\alpha 5$ and the other $\alpha$ subunits in the presence and absence of the PAC3-PAC4 heterodimer. The 'Input1' and 'Input2' lanes contained $\mathrm{His}_{6}$-scPAC3/4 and $\alpha 1-\alpha 6$ subunits in the absence and presence of $\alpha 7$, respectively ( $0.5 \mu \mathrm{g}$ each). (b) Interaction between $\alpha 5$ and the adjacent $\alpha$ subunits, $\alpha 4$ and $\alpha 6$. The 'Input3' lane contained $\mathrm{His}_{6}$-scPAC $3 / 4$ and $\alpha 4-\alpha 6$ subunits. The pull-down experiment was also performed using an scPAC3/4 mutant with V77S and K80A substitutions in PAC3. Band assignments were carried out according to Figure S1b.

In yeast, the Pba3-Pba4 heterodimer acts as a matchmaker, reinforcing interactions between the $\alpha 4$ and $\alpha 5$ subunits [17]. The results of our pull-down analysis indicated that the PAC3-PAC4 heterodimer interacts with $\alpha 4, \alpha 5$, and $\alpha 6$, thereby acting as a molecular matchmaker for these proteasomal subunits. These findings suggest that the functional roles and interactions of this assembly chaperone complex with the proteasomal subunits are evolutionally conserved between yeast and humans.

\subsection{Structural Insights into the Mechanisms Underlying PAC3/PAC4-Dependent $\alpha 4-\alpha 5-\alpha 6$ Assembly}

To investigate the structural mechanisms underlying the chaperone-dependent formation of the $\alpha 4-\alpha 5-\alpha 6$ subcomplex, we built a three-dimensional model of the putative quintet complex comprised of PAC3, PAC4, $\alpha 4, \alpha 5$ and $\alpha 6$, using previously-reported crystallographic data. Crystal structures for the PAC3 homodimer [16], domain-swapped PAC4 homodimer [18], and 20S proteasome [21] have been published. In addition, we newly determined a $0.96-\AA ̊$ high-resolution trigonal structure of the 
PAC3 homodimer (Figure S2a). The overall structure of the trigonal form was very similar to that of the tetragonal structure we have previously published, except for a loop comprised of residues 51-61 (Figure S2b), suggesting that it is mobile. Loop flexibility was also observed in the corresponding segment of the yeast ortholog Pba3 in its heterodimer with Pba4 [16] (Figure S3). Our nuclear magnetic resonance (NMR) relaxation data from the human PAC3 homodimer confirmed that the loop is indeed mobile and disordered in solution (Figure S4).

In the quintet-complex models, in addition to the interactions between PAC3-PAC4 and $\alpha 5$, based on the crystal structure of the yeast counterparts [16], the assembly chaperone interacted with the neighboring $\alpha 4$ and $\alpha 6$ subunits (Figure 3a). When the PAC3-4/ $\alpha 4 / \alpha 5 / \alpha 6$ quintet complex model was superimposed onto the crystal structure of 20S CP, PAC3 and PAC4 make steric hindrance with $\beta 6$ and $\beta 5$, respectively, which possibly triggers the release of PAC3-4 from the $\alpha$-ring upon binding of the $\beta$ subunits onto the $\alpha$-ring. A complex model, model A, based on the 2.00- $\AA$ PAC3 structure showed that the mobile loop was turned toward the solvent. Another model based on the $0.96-\AA$ structure, model $^{\circ}$ $\mathrm{B}$, showed that the corresponding loop contacts $\alpha 6$. Apart from interactions involving this mobile loop, intermolecular contacts between the PAC3-PAC4 heterodimer and the proteasomal subunits are almost identical in the two models. Therefore, in the rest of this paper, we discuss the structural basis of the PAC3/PAC4-dependent $\alpha 4-\alpha 5-\alpha 6$ subunit assembly using model B.

(a)

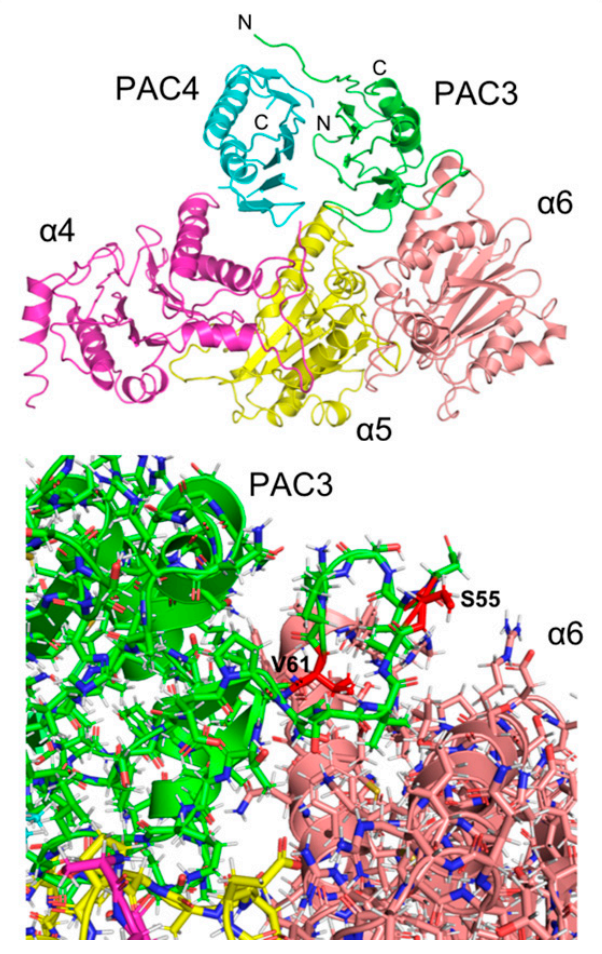

(b)

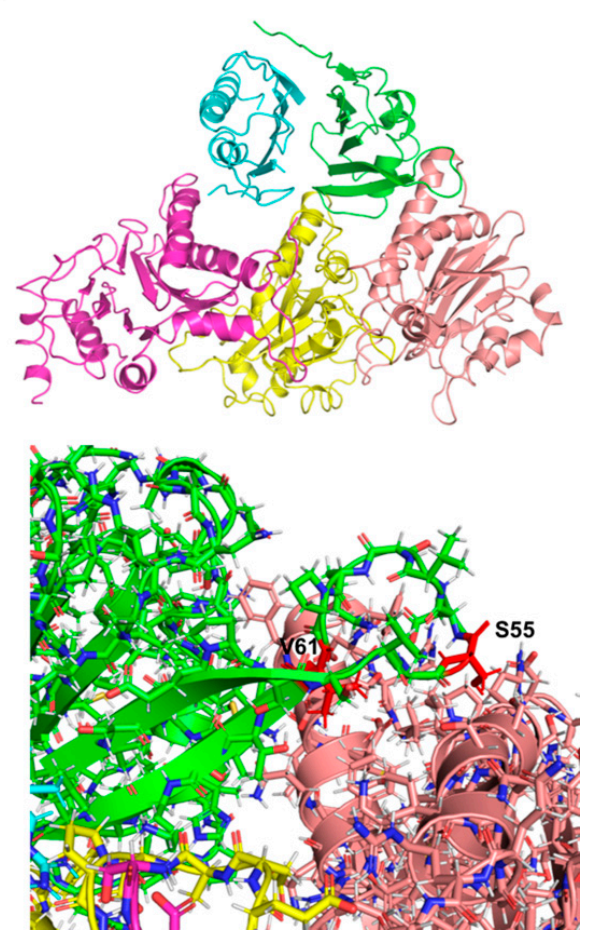

Figure 3. Three-dimensional model of the quintet complex comprising PAC 3, PAC4, $\alpha 4, \alpha 5$, and $\alpha 6$. (a) Complex model A, based on the 2.00- $\AA$ PAC3 structure. (b) Complex model B, based on the newly-determined $0.96-\AA$ structure. The positions of the $\mathrm{N}$ - and C-termini are indicted. Overall and close-up views between PAC 3 and $\alpha 6$ of the quintet-complex models are shown in the upper and lower parts of the figure, respectively. Putative $\alpha 6$-binding residues of PAC3, Ser55 and Val61 (see also Figure $4 b$ ), are highlighted in red in both models to highlight the conformational differences of the loop between the two models.

In this model, the interaction of $\alpha 5$ with the PAC3-PAC4 heterodimer is mediated by Gln70, Glu72, and Lys104 in PAC3, Arg48 in PAC4, and Glu95, His99, Tyr103, and Asp129 in $\alpha 5$ through electrostatic interactions and hydrogen bonds (Figure 4). Our pull-down data indicated that scPAC3/4 interacted most strongly with $\alpha 5$ and weakly with $\alpha 4$ and $\alpha 6$ (Figure 1 ). The model predicted additional 
interactions between PAC3 and $\alpha 6$, and between PAC 4 and $\alpha 4$ (Figure $4 \mathrm{~b}, \mathrm{c}$ ). Specifically, Ser55, Lys80, and Asn81 of PAC3 form hydrogen bonds or electrostatic interactions with Ser110, Asp94, and Arg96 of $\alpha 6$, respectively. There are also predicted hydrophobic interactions of Val61, Phe85, and Val77 in PAC3 with Phe87, Phe97, and Leu93 in $\alpha 6$. Additionally, Asp70 and Arg85 of PAC4 have electrostatic interactions with Arg117 and Glu99 of $\alpha 4$, respectively (Figure 4c). Gln81 and Ile61 of PAC4 form hydrogen bonds and hydrophobic interactions with Ser93 and Val98 of $\alpha 4$, respectively.

To validate our docking model, we performed mutational experiments, especially focusing on the interaction between PAC 3 and $\alpha 6$, which were specifically observed in the human proteins as compared with yeast counterparts. We constructed an scPAC3/PAC4 mutant in which putative $\alpha 6$-binding residues, Val77 and Lys80, of PAC3 are replaced with Ser and Ala, respectively. As expected, our mutational analysis indicated that mutations of Val77 and Lys 80 of PAC3 impaired interaction with $\alpha 6$ but not with $\alpha 4$ and $\alpha 5$ (Figure $2 b$ ), confirming the validity of our docking model.

(a)

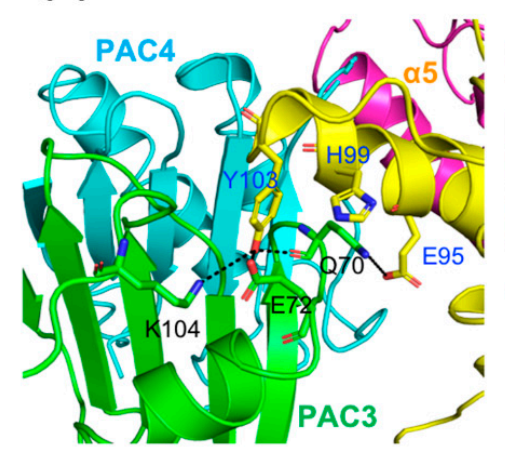

(b)

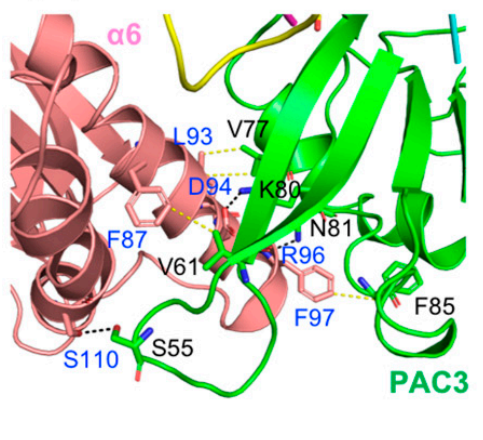

(c)

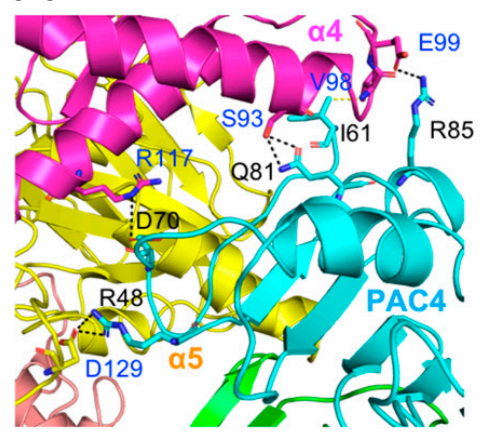

Figure 4. Predicted interaction interfaces between the PAC3-PAC4 heterodimer and proteasomal $\alpha 4-\alpha 5-\alpha 6$ subunits. (a) PAC3- $\alpha 5$. (b) PAC3- $\alpha 6$. (c) PAC4- $\alpha 4$ or $\alpha 5$ interfaces. Residues involved in the interactions are shown as stick representations. Potential hydrogen bonds and non-polar interactions are indicated as black and yellow dotted lines, respectively.

Although the overall structures of human PAC3 and PAC4 are similar to those of yeast Pba3 and Pba4 (RMSD = 1.9-2.1 and 1.9-2.2 $⿱$ ) respectively, their amino acid sequence similarities are low (PAC3 versus Pba3 11.0\%; PAC4 versus Pba $14.6 \%$ ). The $\alpha$-subunit contacting residues of human PAC3 and PAC4, as predicted by the model, are not well-conserved in the yeast orthologs $\mathrm{Pba} 3$ and Pba4 (Figure S5). Nevertheless, our model predicts that the complementarity at the interaction interfaces between the PAC3-PAC4 heterodimer and the proteasomal $\alpha 4-\alpha 5-\alpha 6$ subunits can be conserved in the yeast counterparts with a few exceptions. Perhaps the best example is the replacement of electrostatic interactions between Glu72 of PAC 3 and His 99 of $\alpha 5$ by non-polar contacts between Ala105 of Pba3 and Gln114 of $\alpha 5$. Therefore, despite the low sequence similarity, the overall interaction modes of the matchmaking chaperones with the proteasomal subunits appear to be conserved between humans and yeast. It is plausible that the conformational flexibility of the mobile 51-61 loop of PAC3, which carries the $\alpha 6$-contacting residues, contributes to the interaction adjustability.

In summary, we produced structural insights into the functional mechanisms of the PAC3-PAC4 heterodimer as a molecular matchmaker underpinning the $\alpha 4-\alpha 5-\alpha 6$ subcomplex during $\alpha$-ring formation. These findings offer potential new approaches to the design of inhibitors against the protein-protein interactions involved in proteasome biogenesis.

\section{Methods}

\subsection{Sample Preparation}

Human proteasome $\alpha 6$ short isoform and $\alpha 7$ subunits were produced and purified as previously described [22,23]. Genes encoding the proteasome $\alpha 1$ and $\alpha 4$ subunits were subcloned into NdeI 
and SalI sites in pET28b, and the $\alpha 2$ gene was inserted into the pRSFDuet- 1 vector using NdeI and XhoI restriction enzyme sites (Merck Millipore, Burlington, MA, USA). As for $\alpha 4$, 3xFLAG sequence (DYKDHDGDYKDHDIDYKDDDDK) was added at the N-terminus. The $\alpha 3$ and $\alpha 5$ genes were subcloned into the BamHI and XhoI or SalI sites of modified pCold-I and pCold-GST vectors (TaKaRa Bio Inc., Kusatsu, Japan), respectively, in which a factor Xa cleavage site was replaced with that of TEV protease. The PAC3 and PAC4 genes were subcloned into NdeI and XhoI sites in pET28b, in which the C-terminus of PAC4 was connected to the N-terminus of PAC3 through a (GGGS) 4 liner. Standard polymerase chain reaction method was used to generate a V77S/K80A PAC3 mutant. Escherichia coli BL21-CodonPlus (DE3)-RIL (Agilent Technologies, Santa Clara, CA, USA) was used for all recombinant protein expression.

For the expression of recombinant proteins, the E. coli cells were grown in LB medium containing kanamycin or ampicillin. Briefly, the recombinant proteins were purified from the soluble fractions, except for $\alpha 2$, which was purified from the inclusion bodies and refolded using standard dilution methods.

Purification of these recombinant proteins was performed using affinity chromatography with Anti-FLAG M2 Affinity gel (Sigma-Aldrich, St. Louis, MO, USA), $\mathrm{Ni}^{+}$-charged Chelating Sepharose, or Glutathione Sepharose 4B, anion-exchange chromatography with RESOURCE Q resin, and size exclusion chromatography with Superdex 75 pg or 200 pg resins (GE Healthcare, Chicago, IL, USA). For NMR analyses, the PAC3 homodimer was expressed in E. coli cells which were grown in M9 minimal medium containing $\left[{ }^{13} \mathrm{C}\right]$ glucose $(2.0 \mathrm{~g} / \mathrm{L})$ and/or $\left[{ }^{15} \mathrm{~N}^{-N} \mathrm{Cl}_{4} \mathrm{Cl}(1.0 \mathrm{~g} / \mathrm{L})\right.$, and purified using a previously-described protocol [12].

\subsection{Pull-Down Experiments}

The 3xFLAG-tagged $\alpha 4$, GST-fused proteasome $\alpha 5$-subunit (GST- $\alpha 5$ ), non-tagged or His 6 -tagged forms of proteasome $\alpha 1, \alpha 2, \alpha 3, \alpha 6$, and $\alpha 7$ subunits, and scPAC3/4 were used in the pull-down assays. For immobilization, $20 \mu \mathrm{g}$ of His $_{6}$-tagged scPAC3/4 or GST- $\alpha 5$ was applied to $\mathrm{Ni}^{2+}$-charged Chelating Sepharose or Glutathione Sepharose 4B (GE Healthcare) resins, respectively. The $\mathrm{His}_{6}$-scPAC3/4-immobilized resins were incubated with $50 \mu \mathrm{g}$ of $\alpha 1-\alpha 7$ subunits for $2 \mathrm{~h}$ at $4{ }^{\circ} \mathrm{C}$ in an incubation buffer ( $20 \mathrm{mM}$ Tris- $\mathrm{HCl}(\mathrm{pH} 8.0)$ and $150 \mathrm{mM} \mathrm{NaCl})$. For $\alpha 5$, the GST- $\alpha 5$-immobilized resins were incubated with $50 \mu \mathrm{g}$ of $\alpha 1-\alpha 4, \alpha 6$, and $\alpha 7$ in the presence and absence of $50 \mu \mathrm{g}$ of scPAC $3 / 4$ as described above. Since $\alpha 7$ makes a stable complex with $\alpha 6[19,20]$, the pull-down experiments containing $\alpha 7$ were performed separately. The resins were washed four times with the incubation buffer, which contains $60 \mathrm{mM}$ imidazole in the $\mathrm{His}_{6}$-tag pull-down assays. Proteins bound to the Chelating or Glutathione Sepharose resins were eluted using $20 \mathrm{mM}$ Tris- $\mathrm{HCl}(\mathrm{pH}$ 8.0)/500 mM imidazole or $50 \mathrm{mM}$ Tris- $\mathrm{HCl}(\mathrm{pH} 8.0) / 10 \mathrm{mM}$ reduced glutathione, respectively, and analyzed by SDS-PAGE, stained with CBB.

\subsection{Crystallization, X-ray Data Collection, and Structure Determination}

For crystallization, purified non-tagged PAC3 homodimer was produced at a concentration of $8.0 \mathrm{mg} / \mathrm{mL}$ in $20 \mathrm{mM}$ Tris- $\mathrm{HCl}$ (pH 7.5) and $150 \mathrm{mM} \mathrm{NaCl}$. Crystals were obtained in a buffer containing 30\% PEG2000 monomethyl ether and $0.1 \mathrm{M}$ potassium thiocyanate with incubation at $20^{\circ} \mathrm{C}$ for three to four days. Crystals were transferred into the reservoir solution and flash-cooled in liquid nitrogen. Diffraction intensities were integrated using XDS [24] and data scaling was carried out using AIMLESS [25]. The crystals of PAC 3 belonged to space group $P 3_{1} 21$ and diffracted up to a resolution of $0.96 \AA$.

The trigonal structure of PAC3 was solved by the molecular replacement method using MOLREP [26], using the previously-reported tetragonal structure (PDB code 2Z5E) [16] as a search model. Automated model building and manual model fitting to electron density maps were performed using ARP/wARP [27] and COOT [28], respectively. Model refinement was carried out using REFMAC5 [29], and structure validation was conducted using MolProbity [30]. The data collection 
and refinement statistics of the PAC3 homodimer are summarized in Table S1. The molecular graphics were prepared using PyMOL (Schrödinger, New York, NY, USA).

\subsection{Computer-Aided Model Building}

The quintet-complex model comprising PAC $3, \mathrm{PAC} 4, \alpha 4, \alpha 5$, and $\alpha 6$ was created by several rounds of superimpositions using the coordinates of the human PAC3 homodimer (PDB codes, 2Z5E [16], and 6JPT (from this study)), the human PAC4 homodimer (PDB code: 5WTQ) [18], the human 20S proteasome (5LE5) [21], the yeast Pba3-Pba4 heterodimer (2Z5B) [16], and the yeast Pba3-Pba4/ $\alpha 5$ ternary complex (2Z5C) [16]. The human PAC3-PAC4 heterodimer was created by superimposition of PAC3 and PAC4 monomers onto yeast Pba3 and Pba4, respectively. The resulting PAC3-PAC4 model was superimposed onto the yeast Pba3-Pba4 structure complexed with $\alpha 5$. Finally, to make a quintet complex model, the PAC3-PAC4- $\alpha 5$ (yeast) model was superimposed onto the human $\alpha 5$ subunit of the $20 \mathrm{~S}$ proteasome. Subsequent protonation and energy minimization was performed using the CHARMm force field with the Discovery Studio program suite [31] (BIOVIA, San Diego, CA, USA).

\subsection{NMR Spectroscopy}

${ }^{13} \mathrm{C}$ - and ${ }^{15} \mathrm{~N}$-labeled non-tagged PAC3 homodimer $(0.3 \mathrm{mM})$ and ${ }^{15} \mathrm{~N}$-labeled non-tagged PAC3 homodimer $(0.1 \mathrm{mM})$, dissolved in PBS ( $\mathrm{pH}$ 6.8) containing $10 \% \mathrm{D}_{2} \mathrm{O}(\mathrm{v} / \mathrm{v}), 1 \mathrm{mM}$ EDTA, and $0.01 \% \mathrm{NaN}_{3}$, were used for spectral assignment and relaxation experiments. All NMR data were acquired at $303 \mathrm{~K}$ using DMX-500, AVANCE-500, and AVANCE-800 spectrometers equipped with a 5-mm triple-resonance cryogenic probe (Bruker, Billerica, MA, USA). The NMR data were processed using TOPSPIN (Bruker) and NMRPipe [32]. Conventional 3D NMR experiments [33] were carried out for chemical shift assignments of the heteronuclear single-quantum correlation (HSQC) peaks originating from the PAC3 homodimer. Spectral assignments were carried out using SPARKY [34] and CCPNMR [35] software. ${ }^{15} \mathrm{~N}$ relaxation parameters, $T_{1}, T_{2}$, and ${ }^{15} \mathrm{~N}-{ }^{1} \mathrm{H}$ heteronuclear nuclear Overhauser effect (NOE) were obtained at $303 \mathrm{~K}$ using an AVANCE-800 spectrometer and analyzed using the Protein Dynamics software in the Dynamics Center (Bruker).

\subsection{Accession Numbers}

The coordinates and structural factors of the crystal structure of the PAC3 homodimer have been deposited in the Protein Data Bank under accession number 6JPT. Backbone ${ }^{1} \mathrm{H}$ and ${ }^{15} \mathrm{~N}$ chemical shift data of the PAC3 homodimer have been deposited in the Biological Magnetic Resonance Data Bank under accession number 27844.

Supplementary Materials: Supplementary materials can be found at http://www.mdpi.com/1422-0067/20/9/2231/s1.

Author Contributions: T.S. and K.K. conceived and designed the study; T.S. and E.K. performed protein design and preparation. T.S. performed the pull-down and crystallographic experiments; M.Y.-U. and K.O. performed the NMR experiment; K.T. contributed the materials and reagents; T.S. and K.K. mainly wrote the manuscript.

Funding: This work was supported in part by the Grants-in-Aid for Scientific Research (Grant Numbers JP26460051 to E.K. and JP25102008, JP15H02491 to K.K.) and Nanotechnology Platform Program (Molecule and Material Synthesis) from the Ministry of Education, Culture, Sports, Science and Technology, Japan. This work was also supported by the Joint Research by Exploratory Research Center on Life and Living Systems (ExCELLS) (ExCELLS program No. 18-402).

Acknowledgments: We thank Kumiko Hattori, Kiyomi Senda and Yukiko Isono for their help in the preparation of recombinant proteins. We also thank Hirokazu Yagi (Nagoya City University) for his useful discussion. The diffraction data set was collected at Osaka University using BL44XU at SPring-8 and Nagoya University using BL2S1 at Aichi Synchrotron Radiation Center (Japan). We acknowledge the synchrotron beamline staff and Institute of Drug Discovery Science at Nagoya City University for providing the data collection and computational facilities.

Conflicts of Interest: The authors declare that they have no competing financial interests. 


\section{Abbreviations}

$\begin{array}{ll}\text { CBB } & \text { Coomassie Brilliant Blue } \\ \text { CP } & \text { Core particle } \\ \text { GST } & \text { Glutathione } \text { S-transferase } \\ \text { HSQC } & \text { Heteronuclear single-quantum correlation } \\ \text { NMR } & \text { Nuclear magnetic resonance } \\ \text { NOE } & \text { Nuclear Overhauser effect } \\ \text { PAC } & \text { Proteasome-assembling chaperone } \\ \text { RP } & \text { Regulatory particle } \\ \text { SC } & \text { Single-chain } \\ \text { SDS-PAGE } & \text { Sodium dodecyl sulfate polyacrylamide gel electrophoresis }\end{array}$

\section{References}

1. Tanaka, K. The proteasome: Overview of structure and functions. Proc. Jpn. Acad. Ser. B Phys. Biol. Sci. 2009, 85, 12-36. [CrossRef]

2. Baumeister, W.; Walz, J.; Zuhl, F.; Seemuller, E. The proteasome: Paradigm of a self-compartmentalizing protease. Cell 1998, 92, 367-380. [CrossRef]

3. Budenholzer, L.; Cheng, C.L.; Li, Y.; Hochstrasser, M. Proteasome Structure and Assembly. J. Mol. Biol. 2017, 429, 3500-3524. [CrossRef]

4. Collins, G.A.; Goldberg, A.L. The Logic of the 26S Proteasome. Cell 2017, 169, 792-806. [CrossRef] [PubMed]

5. Murata, S.; Yashiroda, H.; Tanaka, K. Molecular mechanisms of proteasome assembly. Nat. Rev. Mol. Cell Biol. 2009, 10, 104-115. [CrossRef]

6. Kish-Trier, E.; Hill, C.P. Structural biology of the proteasome. Annu. Rev. Biophys. 2013, 42, 29-49. [CrossRef]

7. Tomko, R.J., Jr.; Hochstrasser, M. Molecular architecture and assembly of the eukaryotic proteasome. Annu. Rev. Biochem. 2013, 82, 415-445. [CrossRef]

8. Kato, K.; Satoh, T. Structural insights on the dynamics of proteasome formation. Biophys. Rev. 2018, 10, 597-604. [CrossRef]

9. Hirano, Y.; Hayashi, H.; Iemura, S.; Hendil, K.B.; Niwa, S.; Kishimoto, T.; Kasahara, M.; Natsume, T.; Tanaka, K.; Murata, S. Cooperation of multiple chaperones required for the assembly of mammalian $20 \mathrm{~S}$ proteasomes. Mol. Cell 2006, 24, 977-984. [CrossRef]

10. Le Tallec, B.; Barrault, M.B.; Courbeyrette, R.; Guerois, R.; Marsolier-Kergoat, M.C.; Peyroche, A. 20S proteasome assembly is orchestrated by two distinct pairs of chaperones in yeast and in mammals. Mol. Cell 2007, 27, 660-674. [CrossRef] [PubMed]

11. Almond, J.B.; Cohen, G.M. The proteasome: A novel target for cancer chemotherapy. Leukemia 2002, 16, 433-443. [CrossRef]

12. Doi, T.; Yoshida, M.; Ohsawa, K.; Shin-ya, K.; Takagi, M.; Uekusa, Y.; Yamaguchi, T.; Kato, K.; Hirokawa, T.; Natsume, T. Total synthesis and characterization of thielocin B1 as a protein-protein interaction inhibitor of PAC3 homodimer. Chem. Sci. 2014, 5, 1860-1868. [CrossRef]

13. Zhang, X.; Schulz, R.; Edmunds, S.; Kruger, E.; Markert, E.; Gaedcke, J.; Cormet-Boyaka, E.; Ghadimi, M.; Beissbarth, T.; Levine, A.J.; et al. MicroRNA-101 Suppresses Tumor Cell Proliferation by Acting as an Endogenous Proteasome Inhibitor via Targeting the Proteasome Assembly Factor POMP. Mol. Cell 2015, 59, 243-257. [CrossRef]

14. Chen, D.; Frezza, M.; Schmitt, S.; Kanwar, J.; Dou, Q.P. Bortezomib as the first proteasome inhibitor anticancer drug: Current status and future perspectives. Curr. Cancer Drug Targets 2011, 11, 239-253. [CrossRef]

15. Wu, W.; Sahara, K.; Hirayama, S.; Zhao, X.; Watanabe, A.; Hamazaki, J.; Yashiroda, H.; Murata, S. PAC1-PAC2 proteasome assembly chaperone retains the core $\alpha 4-\alpha 7$ assembly intermediates in the cytoplasm. Genes Cells 2018, 23, 839-848. [CrossRef]

16. Yashiroda, H.; Mizushima, T.; Okamoto, K.; Kameyama, T.; Hayashi, H.; Kishimoto, T.; Niwa, S.; Kasahara, M.; Kurimoto, E.; Sakata, E.; et al. Crystal structure of a chaperone complex that contributes to the assembly of yeast $20 \mathrm{~S}$ proteasomes. Nat. Struct. Mol. Biol. 2008, 15, 228-236. [CrossRef] 
17. Takagi, K.; Saeki, Y.; Yashiroda, H.; Yagi, H.; Kaiho, A.; Murata, S.; Yamane, T.; Tanaka, K.; Mizushima, T.; Kato, K. Pba3-Pba4 heterodimer acts as a molecular matchmaker in proteasome $\alpha$-ring formation. Biochem. Biophys. Res. Commun. 2014, 450, 1110-1114. [CrossRef]

18. Kurimoto, E.; Satoh, T.; Ito, Y.; Ishihara, E.; Okamoto, K.; Yagi-Utsumi, M.; Tanaka, K.; Kato, K. Crystal structure of human proteasome assembly chaperone PAC4 involved in proteasome formation. Protein Sci. 2017, 26, 1080-1085. [CrossRef]

19. Ishii, K.; Noda, M.; Yagi, H.; Thammaporn, R.; Seetaha, S.; Satoh, T.; Kato, K.; Uchiyama, S. Disassembly of the self-assembled, double-ring structure of proteasome $\alpha 7$ homo-tetradecamer by alpha6. Sci. Rep. 2015, 5, 18167. [CrossRef]

20. Kozai, T.; Sekiguchi, T.; Satoh, T.; Yagi, H.; Kato, K.; Uchihashi, T. Two-step process for disassembly mechanism of proteasome $\alpha 7$ homo-tetradecamer by $\alpha 6$ revealed by high-speed atomic force microscopy. Sci. Rep. 2017, 7, 15373. [CrossRef]

21. Schrader, J.; Henneberg, F.; Mata, R.A.; Tittmann, K.; Schneider, T.R.; Stark, H.; Bourenkov, G.; Chari, A. The inhibition mechanism of human $20 \mathrm{~S}$ proteasomes enables next-generation inhibitor design. Science 2016, 353, 594-598. [CrossRef]

22. Sugiyama, M.; Hamada, K.; Kato, K.; Kurimoto, E.; Okamoto, K.; Morimoto, Y.; Ikeda, S.; Naito, S.; Furusaka, M.; et al. SANS simulation of aggregated protein in aqueous solution. Nucl. Instrum. Methods Phys. Res. A 2009, 600, 272-274. [CrossRef]

23. Sugiyama, M.; Kurimoto, E.; Yagi, H.; Mori, K.; Fukunaga, T.; Hirai, M.; Zaccai, G.; Kato, K. Kinetic asymmetry of subunit exchange of homooligomeric protein as revealed by deuteration-assisted small-angle neutron scattering. Biophys. J. 2011, 101, 2037-2042. [CrossRef]

24. Kabsch, W. Xds. Acta Crystallogr. D Biol. Crystallogr. 2010, 66, 125-132. [CrossRef]

25. Evans, P.R. An introduction to data reduction: Space-group determination, scaling and intensity statistics. Acta Crystallogr. D Biol. Crystallogr. 2011, 67, 282-292. [CrossRef]

26. Vagin, A.; Teplyakov, A. MOLREP: An automated program for molecular replacement. J. Appl. Crystallogr. 1997, 30, 1022-1025. [CrossRef]

27. Langer, G.; Cohen, S.X.; Lamzin, V.S.; Perrakis, A. Automated macromolecular model building for X-ray crystallography using ARP/wARP version 7. Nat. Protoc. 2008, 3, 1171-1179. [CrossRef]

28. Emsley, P.; Lohkamp, B.; Scott, W.G.; Cowtan, K. Features and development of Coot. Acta Crystallogr. D Biol. Crystallogr. 2010, 66, 486-501. [CrossRef]

29. Murshudov, G.N.; Vagin, A.A.; Dodson, E.J. Refinement of macromolecular structures by the maximum-likelihood method. Acta Crystallogr. D Biol. Crystallogr. 1997, 53, 240-255. [CrossRef]

30. Chen, V.B.; Arendall, W.B., 3rd; Headd, J.J.; Keedy, D.A.; Immormino, R.M.; Kapral, G.J.; Murray, L.W.; Richardson, J.S.; Richardson, D.C. MolProbity: All-atom structure validation for macromolecular crystallography. Acta Crystallogr. D Biol. Crystallogr. 2010, 66, 12-21. [CrossRef]

31. Spassov, V.Z.; Yan, L. A fast and accurate computational approach to protein ionization. Protein Sci. 2008, 17, 1955-1970. [CrossRef] [PubMed]

32. Delaglio, F.; Grzesiek, S.; Vuister, G.W.; Zhu, G.; Pfeifer, J.; Bax, A. NMRPipe: A multidimensional spectral processing system based on UNIX pipes. J. Biomol. NMR 1995, 6, 277-293. [CrossRef] [PubMed]

33. Uekusa, Y.; Mimura, S.; Sasakawa, H.; Kurimoto, E.; Sakata, E.; Olivier, S.; Yagi, H.; Tokunaga, F.; Iwai, K.; Kato, K. Backbone and side chain ${ }^{1} \mathrm{H},{ }^{13} \mathrm{C}$, and ${ }^{15} \mathrm{~N}$ assignments of the ubiquitin-like domain of human HOIL-1L, an essential component of linear ubiquitin chain assembly complex. Biomol. NMR. Assign. 2012, 6, 177-180. [CrossRef] [PubMed]

34. Goddard, T.D.; Koeller, D.G. Sparky, Version 3.0; University of California: San Francisco, CA, USA, 1993.

35. Vranken, W.F.; Boucher, W.; Stevens, T.J.; Fogh, R.H.; Pajon, A.; Llinas, M.; Ulrich, E.L.; Markley, J.L.; Ionides, J.; Laue, E.D. The CCPN data model for NMR spectroscopy: Development of a software pipeline. Proteins 2005, 59, 687-696. [CrossRef] [PubMed]

(C) 2019 by the authors. Licensee MDPI, Basel, Switzerland. This article is an open access article distributed under the terms and conditions of the Creative Commons Attribution (CC BY) license (http://creativecommons.org/licenses/by/4.0/). 